\title{
Fluid limit of the continuous-time random walk with general Lévy jump distribution functions
}

\author{
Á. Cartea \\ Birkbeck, University of London, London, United Kingdom
}

D. del-Castillo-Negrete

Oak Ridge National Laboratory, Oak Ridge, Tennessee 37831-8071, USA

(Received 26 April 2007; revised manuscript received 27 July 2007; published 3 October 2007)

\begin{abstract}
The continuous time random walk (CTRW) is a natural generalization of the Brownian random walk that allows the incorporation of waiting time distributions $\psi(t)$ and general jump distribution functions $\eta(x)$. There are two well-known fluid limits of this model in the uncoupled case. For exponential decaying waiting times and Gaussian jump distribution functions the fluid limit leads to the diffusion equation. On the other hand, for algebraic decaying waiting times $\psi \sim t^{-(1+\beta)}$ and algebraic decaying jump distributions $\eta \sim x^{-(1+\alpha)}$ corresponding to Lévy stable processes, the fluid limit leads to the fractional diffusion equation of order $\alpha$ in space and order $\beta$ in time. However, these are two special cases of a wider class of models. Here we consider the CTRW for the most general Lévy stochastic processes in the Lévy-Khintchine representation for the jump distribution function and obtain an integrodifferential equation describing the dynamics in the fluid limit. The resulting equation contains as special cases the regular and the fractional diffusion equations. As an application we consider the case of CTRWs with exponentially truncated Lévy jump distribution functions. In this case the fluid limit leads to a transport equation with exponentially truncated fractional derivatives which describes the interplay between memory, long jumps, and truncation effects in the intermediate asymptotic regime. The dynamics exhibits a transition from superdiffusion to subdiffusion with the crossover time scaling as $\tau_{c}$ $\sim \lambda^{-\alpha / \beta}$, where $1 / \lambda$ is the truncation length scale. The asymptotic behavior of the propagator (Green's function) of the truncated fractional equation exhibits a transition from algebraic decay for $t \ll \tau_{c}$ to stretched Gaussian decay for $t \gg \tau_{c}$.
\end{abstract}

DOI: 10.1103/PhysRevE.76.041105

PACS number(s): 05.40.Fb, 02.50.Ey, 05.60.-k

\section{INTRODUCTION}

Macroscopic transport models often arise as fluid or continuum limits of particle kinetic descriptions. A well-known example is the diffusion equation describing the probability distribution function of particles exhibiting a Brownian random walk. In this case, the particle kinetic description is based on a Gaussian uncorrelated stochastic process determining the individual particle displacements. A more general description at the kinetic level is provided by the continuum time random walk (CTRW) model $[1,2]$ that extends the Brownian random walk in two ways. First, contrary to the Brownian random walk, where particles are assumed to jump at fixed time intervals, the CTRW model allows the possibility of incorporating waiting times. In addition, the CTRW model allows the possibility of using general, non-Gaussian jump distribution functions with divergent second order moments to account for anomalously large particle displacements known as "Lévy flights."

The fundamental equation for the CTRW is the MontrollWeiss equation [1] that describes the probability distribution function of particle displacements in terms of the waiting time distribution $\psi(t)$ and the jump distribution function $\eta(x)$. However, the Montroll-Weiss master equation contains in a sense too much information which might be irrelevant from the point of view of the long-time behavior of large scale macroscopic transport. This motivates taking the fluid or continuum limit of which two cases have been widely studied. One is the Markovian-Gaussian case which neglects memory effects in the waiting time distribution function and assumes a Gaussian jump distribution function. As expected, both assumptions lead in the fluid limit to the diffusion equation. The other case corresponds to the inclusion of memory effects and long jumps with divergent second-order moments through slowly algebraic decaying waiting time $\psi \sim t^{-(1+\beta)}$ and jump distribution functions $\eta \sim x^{-(1+\alpha)}$ with $0<\beta<1$ and $0<\alpha<2$. As is well known, in this case, the resulting macroscopic transport equation is the fractional diffusion equation of order $\beta$ in time and order $\alpha$ in space (see, for example, Ref. [3], and references therein).

One might be tempted to think that the two cases mentioned above encompass all the fundamentally different separable CTRWs and corresponding fluid limits. After all, in the absence of memory, according to the generalized central limit theorem, the sum of individual particle displacement asymptotically converges to either a Gaussian (described by the diffusion equation) if $\eta(x)$ has a finite second moment or to a Lévy stable distribution (described by the fractional equation) if $\eta(x)$ has a divergent second moment. However, what is missing from this simple view is the fact that although the dynamics asymptotically converge to a Gaussian or a Lévy stable distribution, the convergence rate could be extremely slow. A clear example are the truncated Lévy processes introduced in Refs. [4,5] as a way to eliminate the arbitrary large flights produced by Lévy stable distributions. Since truncated distributions have finite second moments, Gaussian convergence is expected. However, what was observed in Refs. [4-6], and several subsequent studies, is that the convergence rate is so slow that for practical purposes (i.e., for time scales typically considered in applications) the 
process can be considered non-Gaussian but not described by Lévy stable distributions.

Truncated Lévy processes have shown applicability in many areas. In the context of plasma physics it has been shown that truncated Lévy distributions reproduce the probability distribution function of the electrostatic potential fluctuations measured in the edge of ohmically heated tokamaks [7]. In fluids, it was observed that the probability distribution function and scaling properties of truncated Lévy processes display several features of two-dimensional turbulence simulations including a sharp transition from algebraic to exponential decay in the tails of the velocity probability distribution function [8]. In Ref. [9] truncated Lévy distributions were used to fit and model the statistics of interplanetary solar-wind velocity and magnetic velocity fluctuations measured in the heliosphere. Finally, in finance it has been shown that truncated Lévy distributions describe the scaling of the probability distribution function of the S\&P 500 economic index [10].

One of our main motivations for studying truncated Lévy distributions stems from our studies of turbulent transport in magnetically confined plasmas. In this system transport is typically nondiffusive, and it has been shown that CTRW models $[11,12]$ and fractional diffusion transport equations [13-15] are capable of describing some of the basic phenomenology. In particular, in pressure gradient driven plasma turbulence the trapping effects of $\mathbf{E} \times \mathbf{B}$ eddies give rise to waiting time distributions with power law tails and avalanchelike transport events give rise to jump distributions with power law decay in Refs. $[16,13]$. In this system, transport is described by a space-time fractional diffusion equation with $\alpha=3 / 4$ and $\beta=1 / 2$ [14]. However, the transport models considered up to now have been based on the use of Lévy stable distributions which, as discussed before, allow for the possibility of arbitrarily large transport events characterized by divergent second moments. Although the presence of large displacements has been well documented in numerical simulations of turbulent transport, it is clear from the physical point of view that particle displacements cannot be arbitrarily large. In particular, it is expected that some sort of decorrelation in the particles trajectories or boundary effects in finite size systems will eventually lead to the truncation of otherwise arbitrary large transport events. Accordingly, to make further progress in the modeling of nondiffusive turbulent transport it is important to study CTRWs and their associated macroscopic transport for truncated Lévy distributions.

Despite their apparent widespread applicability, little is known about the role of truncated Lévy distributions in CTRW models, and most importantly about the role of the truncation effects in the formulation of macroscopic transport equations with memory. Among the few previous studies on macroscopic transport models incorporating truncated Lévy processes is Ref. [17] where a special case of a distributed order fractional diffusion equation was proposed to describe a power-law truncated Lévy process. In this case, a Lévy distribution with a power law decay of order $1+\alpha$ is truncated by a steeper power law distribution with decay 5 $-\alpha$. Although moments of order higher than $4-\alpha$ still diverge, the (slow) Gaussian convergence is guaranteed by the existence of the second moment. The models discussed here are based on exponentially truncated Lévy distributions which have finite moments of all orders. In addition, we incorporate memory effects which were not addressed in Ref. [17]. In Refs. [18-20] subordination arguments from probability theory were presented for the study of the scaling limit of the CTRW with memory and general Lévy distributions. The resulting generalized transport equation contains a fractional time derivative describing the memory and the infinitesimal generator of a general Lévy process describing the jumps. Here we follow a complimentary approach based on the Montroll-Weiss master equation for a general CTRW and show that the corresponding generalized transport equation naturally arises from a fluid (long wavelength) limit.

One of the main goals of the present work is to study the interplay of memory and truncation effects in fractional diffusion. To address this problem we propose a fractional equation with exponentially truncated fractional derivatives. This equation follows from the generalized transport equation mentioned above. We show that the solution of the exponentially truncated equation exhibits a scaling transition. In particular, the return probability distribution function exhibits a transition from the Lévy stable scaling $G(0, t)$ $\sim t^{-\beta / \alpha}$ at short times, to the scaling $G(0, t) \sim t^{\beta / 2}$ at long times, where $\alpha$ and $\beta$ are the orders of the fractional derivatives in space and time, respectively. For $2 \beta / \alpha>1$ this corresponds to a transition from superdiffusive to subdiffusive dynamics. The crossover time to subdiffusive behavior scales as $\tau \sim \lambda^{-\alpha / \beta}$, where $1 / \lambda$ is the truncation length scale. We also show that the asymptotic behavior of the propagator (Green's function) of the truncated fractional diffusion equation exhibits a transition from algebraic decay (characteristic of superdiffusive processes) for $\lambda^{\alpha / \beta} t \ll 1$ to stretched Gaussian decay (characteristic of subdiffusive processes) for $\lambda^{\alpha / \beta} t \gg 1$, where $1 / \lambda$ is the characteristic scale length of the truncation.

The rest of the paper is organized as follows. The next section discusses the fluid limit of the Montroll-Weiss master equation for a separable CTRW with memory and a general Lévy stochastic process. Section III focuses on the study of exponentially truncated processes. The corresponding fractional diffusion equation is formulated and applications including scaling transitions are presented. The conclusions and a summary of the results are presented in Sec. IV.

\section{FLUID LIMIT OF GENERAL, SEPARABLE CTRWs}

The CTRW model consists of an ensemble of particles that at times $t_{1}, t_{2}, \ldots, t_{i}, \ldots$, experience a displacement, or jump, $x_{1}, x_{2}, \ldots, x_{i}, \ldots$. Both the waiting times $\tau_{i}=t_{i}-t_{i-1}$ and the jumps $x_{i}$ are random variables drawn from a waitingtime distribution $\psi(\tau)$ and a jump distribution $\eta(x)$, respectively. Given $\eta$ and $\psi$, the probability of finding a particle at position $x$ and time $t$ is determined by the Montroll-Weiss (MW) equation $[1,21,3]$ which in Fourier-Laplace space takes the form

$$
\hat{\widetilde{P}}(k, s)=\frac{1-\tilde{\psi}(s)}{s} \frac{1}{1-\tilde{\psi}(s) \hat{\eta}(k)},
$$

where $\mathcal{L}\{\psi\}=\widetilde{\psi}(s)=\int_{0}^{\infty} e^{s t} \psi(t) d t$ is the Laplace transform of the waiting time distribution function and $\hat{\eta}(k)$ 
$=\int_{-\infty}^{\infty} e^{i k x} \eta(x) d x$ is the Fourier transform of the jump distribution function. Introducing the survival probability distribution function

$$
\Psi=\int_{t}^{\infty} \psi\left(t^{\prime}\right) d t^{\prime}, \quad \widetilde{\Psi}=\frac{1-\tilde{\psi}}{s},
$$

Eq. (1) becomes

$$
\widetilde{\hat{P}}=\frac{\widetilde{\Psi}}{1-[1-s \widetilde{\Psi}] \hat{\eta}} .
$$

Following Refs. [22,23] we consider $\Psi(t)=E_{\beta}\left(-t^{\beta}\right)$, where $E_{\beta}$ is the Mittag-Leffler function for which (see, for example, Ref. [24])

$$
\mathcal{L}\left\{t^{n \beta} E_{\beta}^{(n)}\left[ \pm a t^{\beta}\right]\right\}=\frac{n ! s^{\beta-1}}{\left(s^{\beta} \mp a\right)^{m+1}}, \quad \operatorname{Re}(s)>|a|,
$$

where $E_{\beta}^{(n)}(z)=\frac{d^{n}}{d z^{n}} E_{\beta}(z)$. Using Eq. (4), the Montroll-Weiss equation reduces to

$$
\tilde{\hat{P}}=\frac{s^{\beta-1}}{s^{\beta}+1-\hat{\eta}} .
$$

Introducing the Caputo fractional derivative in time

$$
{ }_{0}^{c} D_{t}^{\beta} f=\frac{1}{\Gamma(1-\beta)} \int_{0}^{t} \frac{\partial_{\tau} f}{(t-\tau)^{\beta}} d \tau, \quad \mathcal{L}\left[{ }_{0}^{c} D_{t}^{\beta} f\right]=s^{\beta} \tilde{f}-s^{\beta-1} f(0),
$$

Eq. (5) yields for $P(x, t=0)=\delta(x)$

$$
{ }_{0}^{c} D_{t}^{\beta} \hat{P}(k, t)=[\hat{\eta}-1] \hat{P} .
$$

The use of the Mittag-Leffler function allowed the exact inversion of the Laplace transform in terms of the fractional time derivative, something that is not possible for a general waiting time distribution function. However, in the fluid limit what matters is the asymptotic decay of the survival probability distribution function. Thus, any distribution that exhibits the same algebraic decay as the Mittag-Leffler function, namely, $\psi \sim t^{-(\beta+1)}$ will yield Eq. (7) in the fluid limit. What is remarkable about the Mittag-Leffler function is that, as originally pointed out in Refs. [22,23], this choice leads directly to a fractional equation in time without the need to take a time asymptotic $(s \rightarrow 0)$ limit. Note that, as expected, since $E_{1}(z)=e^{z}$, the inversion is also exact in the Markovian $\beta=1$ case.

In Fourier space, the large scale macroscopic fluid limit corresponds to $k \rightarrow 0$. In this limit, approximating $\hat{\eta}(k)$ $=e^{\Lambda(k)} \approx 1+\Lambda(k)+\ldots$ in Eq. (7) leads to

$$
{ }_{0}^{c} D_{t}^{\beta} \hat{P}(k, t)=\Lambda \hat{P},
$$

where $\Lambda$ is the characteristic exponent of $\eta$, the probability distribution for jumps. Equation (8) is a macroscopic transport equation describing, in the small $k$ fluid limit, the dynamics of a CTRW with a general probability distribution function of jumps with characteristic function $\hat{\eta}=e^{\Lambda}$. As we will discuss in the next section, this equation contains as a special case the well-known fractional diffusion equation corresponding to Lévy stable distributions but, most importantly, it allows the incorporation in the fluid limit of more general stochastic process including truncated Lévy distribution functions.

Using Eqs. (4) and (6) it is straightforward to show that the general solution of Eq. (8) for an initial condition $P_{0}(x)$ $=P(x, t=0)$ is

$$
P(x, t)=\frac{1}{2 \pi} \int_{-\infty}^{\infty} G(x-y, t) P_{0}(y) d y,
$$

where the Green's function or propagator $G$ is given by

$$
G(x, t)=\frac{1}{2 \pi} \int_{-\infty}^{\infty} e^{-i k x} E_{\beta}\left[t^{\beta} \Lambda(k)\right] d k .
$$

The form of Eq. (10) is similar to the form of the solution of the standard fractional diffusion equation, see, for example, Refs. $[25,26]$. However, it is important to keep in mind that Eq. (10) is more general since, contrary to the well-studied standard case, the function $\Lambda$ is not restricted to be of the $\alpha$-stable type. For a probabilistic interpretation of the solution in Eq. (10) based on subordinated processes see Refs. $[18,19]$. Note that in the Markovian $\beta=1$ case, $E_{1}(z)=e^{z}$ and the Fourier transform of the propagator of the macroscopic transport equation at time $t, \hat{G}(k, t)=e^{t \Lambda}=(\hat{\eta})^{t}$, is the $t$ power of the characteristic function of the "microscopic" particle jump distribution function.

To make further progress we need to specify the form of the probability distribution function of jumps $\eta$. Here we assume that this function belongs to the large class of infinitely divisible distributions. The logarithm of the corresponding characteristic function $\hat{\eta}$ is given by the LévyKhintchine representation [27]

$$
\Lambda=\ln \hat{\eta}=\operatorname{aik}-\frac{1}{2} \sigma^{2} k^{2}+\int_{-\infty}^{\infty}\left[e^{i k x}-1-i k u(x)\right] w(x) d x,
$$

where $a$ is a constant and $\sigma \geq 0$. The function $u(x)$ is a truncation function used to remove the singularity of the integrand at the origin and to guarantee the convergence of the integral. It can be shown that the specific form of this function is irrelevant and that different choices only manifest as different rescalings of the constant $a$. The function $w(x)$ is called the Lévy density and it satisfies $\int \min \left\{1, x^{2}\right\} w(x) d x$ $<\infty$. Substituting Eq. (11) into Eq. (8) and taking the inverse Fourier transform yields

$$
\begin{aligned}
{ }_{0}^{c} D_{t}^{\beta} P= & -a \partial_{x} P+\frac{1}{2} \sigma^{2} \partial_{x}^{2} P+\int_{-\infty}^{\infty}[P(x-y, t)-P(x, t) \\
& \left.+u(y) \partial_{x} P\right] w(y) d y .
\end{aligned}
$$

Equation (12) is the macroscopic transport equation describing the continuum, fluid limit of a CTRW with a general jump distribution function $\eta$ characterized by a general Lévy density $w(y)$. For an alternative derivation of Eq. (12) see Refs. [18-20]. As expected, the integral operator on right- 
hand side of Eq. (12) is the infinitesimal generator of a general Lévy process. By a general Lévy process we mean a stochastic process $x_{t}$, for $0<t<\infty$ and $x_{0}=0$ consisting of independent and stationary increments with logcharacteristic function $t \Lambda$ with $\Lambda$ defined in Eq. (11) [27]. According to this definition a Lévy process consists of a combination of a drift component, a Brownian motion (Gaussian) component, and a jump component. These three components are completely determined by the LévyKhintchine triplet $\left(a, \sigma^{2}, w\right)$. The constant $a$ parametrizes the "trend" component which is responsible for the development of the process $x_{t}$ on the average. The parameter $\sigma^{2}$ defines the variance of the continuous Gaussian component of $x_{t}$. Finally, the Lévy density $w$ is responsible for the behavior of the jump component of $x_{t}$ and determines the frequency and magnitude of jumps. Given the fact that the propagator in Eq. (10) gives already the solution of the fluid limit of the CTRW, one might wonder about the usefulness of Eq. (12). The key issue here is that having Eq. (12) is conceptually important when studying the role of general Lévy processes (and, in particular, truncated processes) in other problems for which Eq. (10) is not necessary the solution. Two important examples would be the study of the role of truncation effects in the fractional Fokker-Plank equation or in the propagation of fronts in the fractional Fisher-Kolmogorov equation. In principle, such studies could be carried out by adding to Eq. (12) an external potential in the Fokker-Planck case or the corresponding reaction kinetics in the Fisher-Kolmogorov case.

\section{APPLICATION: FRACTIONAL DIFFUSION EQUATION FOR EXPONENTIALLY TRUNCATED LÉVY PROCESSES}

In this section we consider two specific cases of Lévy processes and obtain from Eq. (12) the corresponding macroscopic transport equations. The first example corresponds to the Lévy stable case and recovers the well-known derivation of the standard fractional diffusion equation [3,23]. The second example considers the case of an exponentially truncated Lévy process and leads to a fractional transport equation describing the interplay of memory effects, long-range jumps, and truncation.

For a Lévy stable process the Lévy density is given by

$$
w_{\mathrm{LS}}(x)= \begin{cases}c \frac{(1+\theta)}{2}|x|^{-(1+\alpha)} & \text { for } x<0, \\ c \frac{(1-\theta)}{2} x^{-(1+\alpha)} & \text { for } x>0,\end{cases}
$$

where $c>0$ and $-1 \leq \theta \leq 1$ is an asymmetry parameter. Substituting Eq. (13) into Eq. (11) and integrating, it follows that

$$
\begin{aligned}
\Lambda_{\mathrm{LS}}= & i a k-\frac{1}{2} \sigma^{2} k^{2} \\
& -\left\{\begin{array}{c}
c|k|^{\alpha}\{1+i \theta \operatorname{sgn}(k) \tan (\alpha \pi / 2)\} \\
c|k|\left\{1+\frac{2 i \theta}{\pi} \operatorname{sgn}(k) \ln |k|\right\}
\end{array} \quad \text { for } \alpha \neq 1,\right.
\end{aligned}
$$

where $\operatorname{sgn}(k)=|k| / k$. As expected, $\Lambda_{\mathrm{LS}}$ is the characteristic exponent for a Lévy stable process. Note, however, that we are using a different sign convention for the asymmetry compared to the one used in Ref. [28].

Introducing the Riemann-Liouville fractional derivatives $[24,29]$

$$
\begin{aligned}
& { }_{a} D_{x}^{\alpha} f=\frac{1}{\Gamma(m-\alpha)} \frac{\partial^{m}}{\partial x^{m}} \int_{a}^{x} \frac{f(y)}{(x-y)^{\alpha+1-m}} d y, \\
& { }_{x} D_{b}^{\alpha} f=\frac{(-1)^{m}}{\Gamma(m-\alpha)} \frac{\partial^{m}}{\partial x^{m}} \int_{x}^{b} \frac{f(y)}{(y-x)^{\alpha+1-m}} d y,
\end{aligned}
$$

with $m-1 \leq \alpha<m$ and using

$$
\mathcal{F}\left[{ }_{-\infty} D_{x}^{\alpha} f\right]=(-i k)^{\alpha} \hat{f}, \quad \mathcal{F}\left[{ }_{x} D_{\infty}^{\alpha} f\right]=(i k)^{\alpha} \hat{f},
$$

Eq. (8) for $\Lambda=\Lambda_{\mathrm{LS}}$ gives the well-known fractional diffusion equation

$$
{ }_{0}^{c} D_{t}^{\beta} P(x, t)=-a \partial_{x} P+\frac{1}{2} \sigma^{2} \partial_{x}^{2} P+c\left[l_{-\infty} D_{x}^{\alpha}+r_{x} D_{\infty}^{\alpha}\right] P,
$$

where the weighting factors are defined as

$$
l=-\frac{(1-\theta)}{2 \cos (\alpha \pi / 2)}, \quad r=-\frac{(1+\theta)}{2 \cos (\alpha \pi / 2)} .
$$

\section{A. Exponentially truncated Lévy distribution}

For exponentially truncated Lévy processes [5] the Lévy density is given by

$$
w_{\mathrm{ET}}(x)= \begin{cases}c \frac{(1+\theta)}{2}|x|^{-(1+\alpha)} e^{-\lambda|x|} & \text { for } x<0, \\ c \frac{(1-\theta)}{2} x^{-(1+\alpha)} e^{-\lambda x} & \text { for } x>0,\end{cases}
$$

$0<\alpha \leq 2, c>0,-1 \leq \theta \leq 1$, and $\lambda \geq 0$. In this case the integral in Eq. (11) leads to the characteristic exponent [5]

$$
\Lambda_{\mathrm{ET}}=i a k-\frac{\sigma^{2}}{2} k^{2}-\frac{c}{2 \cos (\alpha \pi / 2)}\left\{\begin{array}{l}
(1+\theta)(\lambda+i k)^{\alpha}+(1-\theta)(\lambda-i k)^{\alpha}-2 \lambda^{\alpha}, \\
(1+\theta)(\lambda+i k)^{\alpha}+(1-\theta)(\lambda-i k)^{\alpha}-2 \lambda^{\alpha}-2 i k \alpha \theta \lambda^{\alpha-1},
\end{array}\right.
$$


for $0<\alpha<1$ and $1<\alpha \leq 2$, respectively. The interpretation of the parameters is very similar to the Lévy stable case. The damping parameter $\lambda$ can be seen as the "strength" of the exponential decay of the tails. When $\lambda \rightarrow 0$, the further out in the tails of the distribution the dampening will take effect and the longer the process will take to become Gaussian as a consequence of the central limit theorem. Moreover, it is straightforward to observe that for $\lambda>0$ the process will have finite moments of all orders. Substituting Eq. (21) into Eq. (8) and using

$$
\begin{array}{r}
\mathcal{F}\left[e^{-\lambda x}{ }_{-\infty} D_{x}^{\alpha} e^{\lambda x} P\right]=(\lambda-i k)^{\alpha} \hat{P}, \\
\mathcal{F}\left[e^{\lambda x}{ }_{x} D_{\infty}^{\alpha} e^{-\lambda x} P\right]=(\lambda+i k)^{\alpha} \hat{P}
\end{array}
$$

we obtain, after inverting the Fourier transform,

$$
{ }_{0}^{c} D_{t}^{\beta} P=-V \partial_{x} P+\frac{\sigma^{2}}{2} \partial_{x}^{2} P+c \mathcal{D}_{x}^{\alpha, \lambda} P-\nu P,
$$

where the $\lambda$-truncated fractional derivative operator of order $\alpha, \mathcal{D}_{x}^{\alpha, \lambda}$ is defined as

$$
\mathcal{D}_{x}^{\alpha, \lambda}=l e^{-\lambda x}{ }_{-\infty} D_{x}^{\alpha} e^{\lambda x}+r e^{\lambda x} D_{\infty}^{\alpha} e^{-\lambda x},
$$

where $V=a$ for $0<\alpha<1$ and $V=a-v$ for $1<\alpha<2$ with

$$
v=\frac{c \alpha \theta \lambda^{\alpha-1}}{|\cos (\alpha \pi / 2)|}
$$

and

$$
\nu=-\frac{c \lambda^{\alpha}}{\cos (\alpha \pi / 2)} .
$$

As expected, for $\lambda=0$ Eq. (23) reduces to Eq. (18).

\section{B. Crossover and slow convergence to subdiffusive transport}

As an application of Eq. (23) we consider the interplay between memory effects and truncation. In particular, we show that for $\beta \neq 1$, in the presence of a truncated Lévy process, the probability of first return exhibits a transition from superdiffusive dynamics to subdiffusive dynamics. We also show that this transition manifests as a crossover from algebraic decaying tails to exponentially decaying tails in the probability distribution function.

For $a=0$ and $\sigma=0$, the characteristic exponent in Eq. (21) satisfies the scaling relation $\Lambda_{\mathrm{ET}}(\mu k ; \mu \lambda)=\mu^{\alpha} \Lambda_{\mathrm{ET}}(k ; \lambda)$. Using Eq. (10), this scaling implies the following scaling for the propagator $G$ :

$$
G(x, \mu t ; \lambda)=\mu^{-\beta / \alpha} G\left(\mu^{-\beta / \alpha} x, t ; \mu^{\beta / \alpha} \lambda\right) .
$$

Note that due to the dependence on $\lambda$ there is no space-time self-similarity, physically the truncation introduces the preferred length scale $1 / \lambda$ that breaks the scale invariance. That is, the probability distribution function at a time $\mu t$ cannot be obtained from a simple rescaling in $x$ of the probability distribution function at $t$, unless $\lambda$ is also rescaled.

To gain some intuition into the role of truncation consider the moments of the propagator

$$
\left\langle x^{n}\right\rangle=\int_{-\infty}^{\infty} x^{n} G(x, t) d x=\left.(-i)^{n} \frac{\partial^{n} \hat{G}(k, t)}{\partial k^{n}}\right|_{k=0}
$$

where according to Eq. (10), $\hat{G}=E_{\beta}\left[t^{\beta} \Lambda_{\mathrm{ET}}\right]$. For $\lambda=0$, only the moments of order $n<\alpha$ exist, which, as discussed before, is one of the drawbacks of the Lévy-Stable distributions from the applications point of view. On the other hand, for $\lambda \neq 0$, the function $\Lambda_{\mathrm{ET}}(k)$ is $C^{\infty}$ at $k=0$, and all the moments of the distribution exist. The first-order moment exhibits the usual scaling

$$
\langle x\rangle=\frac{V}{\Gamma(\beta+1)} t^{\beta},
$$

where $V=a-v$ for $0<\alpha<1$, and $V=a$ for $1<\alpha<2$, with $v$ defined in Eq. (25). Here the truncation plays no role in the scaling exponent. However, note that depending on the values of $\alpha$ and the asymmetry parameter $\theta$, the truncation can increase or decrease the drift velocity $a$. For the time evolution of the variance the second moment gives

$$
\begin{aligned}
\sigma^{2}= & \left\langle[x-\langle x\rangle]^{2}\right\rangle=\left[\frac{2}{\Gamma(2 \beta+1)}-\frac{1}{\Gamma^{2}(\beta+1)}\right] V^{2} t^{2 \beta} \\
& +\frac{2 \chi}{\Gamma(\beta+1)} t^{\beta},
\end{aligned}
$$

where we have introduced the effective diffusivity

$$
\chi=\frac{\sigma^{2}}{2}+\frac{c \alpha|\alpha-1|}{2|\cos (\alpha \pi / 2)| \lambda^{2-\alpha}} .
$$

The truncation increases the Gaussian diffusivity $\sigma^{2} / 2$ by a term inversely proportional to $\lambda$ and directly proportional to $c$, the strength of the truncated Lévy density in Eq. (20). Furthermore, in the limit $\lambda \rightarrow 0, \chi \rightarrow \infty$, a result consistent with the divergence of the second moment in the absence of truncation.

Of particular interest is the scaling in time of the probability of first return $G(x=0, t)$. From the scaling relation in Eq. (27) we have $G(0, t ; \lambda)=t^{-\beta / \alpha} G\left(0,1 ; t^{\beta / \alpha} \lambda\right)$. In the absence of truncation $\lambda=0$, this implies the expected scaling $G(0, t) \sim t^{-\beta / \alpha}$ which in the case $\alpha=2, \beta=1$, reduces to the well-known diffusive scaling $G(0, t) \sim t^{-1 / 2}$. The scaling $G(0, t) \sim t^{-\beta / \alpha}$ remains valid for $\lambda \neq 0$ and small enough times because $\lim _{t \rightarrow 0} G\left(0,1 ; t^{\beta / \alpha} \lambda\right)=G(0,1 ; 0)$. The longtime behavior is less trivial and leads to a different scaling. The decay of the Mittag-Leffler function implies that in the limit $t \rightarrow \infty$ the maximum contribution to the integral in Eq. (10) comes from the region around $k \sim 0$. Therefore, using stationary phase arguments we can get the leading order asymptotic behavior by Taylor expanding the argument of the Mittag-Leffler function around $k=0$. Note, however, that some care must be taken since, contrary to the usual situation involving exponentials, $E_{\beta}$ decays algebraically for $\beta \neq 1$. Restricting attention to the symmetric case $\theta=0$ with no drift $a=0$, and substituting the small $k$ expansion $\Lambda_{\mathrm{ET}}=-\chi k^{2}+\ldots$ into Eq. (10) we get 


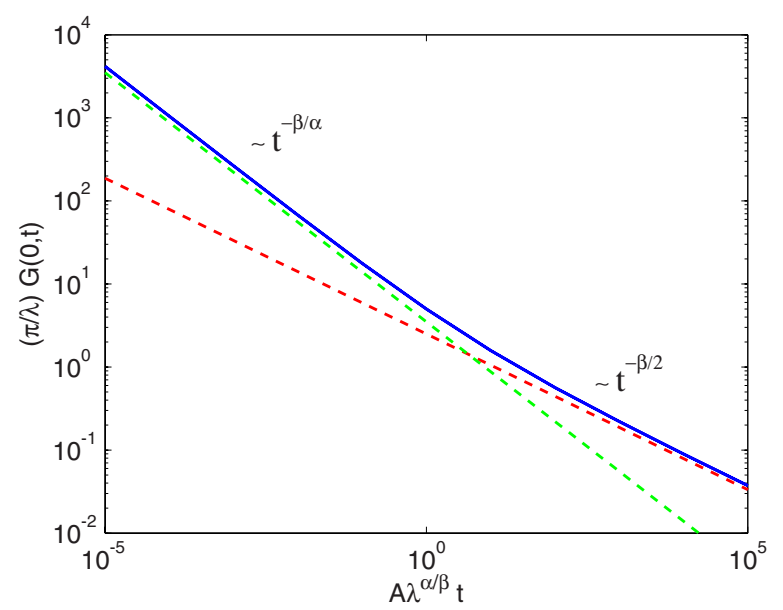

FIG. 1. (Color online) Probability of first return $G(0, t)$ for the truncated fractional diffusion Eq. (23) obtained from the numerical evaluation of the general solution in Eq. (10) for $\Lambda=\Lambda_{\mathrm{ET}}$ with $\theta$ $=0, \alpha=1.25$, and $\beta=0.75$. The solid line denotes the value of $G(0, t)$, the steeper dashed line corresponds to the superdiffusive scaling $G(0, t) \sim t^{-\beta / \alpha}$, and the less steep dashed line corresponds to the subdiffusive scaling $G(0, t) \sim t^{-\beta / 2}$. The scaling constant in the horizontal axis is $A=[c /|\cos (\alpha \pi / 2)|]^{1 / \beta}$.

$$
\begin{aligned}
\lim _{t \rightarrow \infty} G(0, t ; \lambda) & \sim \frac{1}{\pi} \int_{0}^{\infty} E_{\beta}\left[-\chi t^{\beta} k^{2}\right] d k \\
& =\left[\frac{1}{\pi \sqrt{\chi}} \int_{0}^{\infty} E_{\beta}\left(-u^{2}\right) d u\right] t^{-\beta / 2} .
\end{aligned}
$$

Thus, the truncation gives rise to a transition in the scaling of the return probability from $\sim t^{-\beta / \alpha}$ for $t \ll 1$ to $\sim t^{-\beta / 2}$ for $t \gg 1$. In the case $2 \beta / \alpha>1$ this corresponds to a transition from superdiffusive scaling to subdiffusive scaling. This transition is clearly observed in Fig. 1 which shows the return probability as a function of time over ten orders of magnitude obtained from the direct numerical evaluation of the integral in Eq. (10) with $\alpha=1.25, \beta=0.75$, and $\theta=0$. From the scaling of the probability distribution in Eq. (27) it follows that the time $\tau_{c}$ for the crossover to subdiffusive dynamics scales as

$$
\tau_{c} \sim c^{-1 / \beta} \lambda^{-\alpha / \beta}
$$

As expected, in the Markovian case, $\beta=1$, we recover the scaling for ultraslow Gaussian convergence reported in Refs. $[4,6]$.

For symmetric $\theta=0$ processes with $1<\alpha<2$ and $a=\sigma$ $=0$, the propagator in Eq. (10) can be written as

$$
(\pi / \lambda) G(\lambda x, \tau)=\int_{0}^{\infty} \cos (\lambda x u) E_{\beta}\left[\tau^{\beta} \Phi(u)\right] d u,
$$

where

$$
\Phi(u)=\left(1+u^{2}\right)^{\alpha / 2} \cos \left[\alpha \tan ^{-1}(u)\right]-1
$$

and

$$
\tau=[-c / \cos (\alpha \pi / 2)] \lambda^{\alpha / \beta} t .
$$

Figure 2 shows the temporal evolution of the propagator $G$ as a function of $x$ obtained from the numerical evaluation of Eq. (34) for $\alpha=1.25$ and $\beta=0.75$. Because of the truncation, the evolution is not self-similar. In particular, the solution exhibits a transition in (log-normal scale) from a concave profile shape at short times to a convex shape at long times. At the crossover time scale $\tau \sim 1$ the distribution exhibits a linear profile indicating exponential decay. To ease the comparison at different times we have rescaled the spatial dimension by $\sigma_{\beta}=t^{\beta / 2}$, which corresponds to the scaling of the variance in the subdiffusive limit. To maintain the normalization of the probability, the vertical axis has been rescaled by $\sigma_{\beta}$. Figure 2(a) shows the propagator in the small $\tau$ limit. As expected, in this case $G$ exhibits the characteristic form of the Green's function of the fractional diffusion equation for $\beta<1<\alpha<2$. The superimposed dashed line shows the function $G_{\alpha, \beta}^{\infty}(z)=z^{-(1+\alpha)}$. It is observed that for $\lambda x / \sigma \gg 1, G$ $\rightarrow G_{\alpha, \beta}^{\infty}$. This result is expected because of the fact that for small $\tau$ the truncation effects are negligible and the tails of $G$ exhibit the algebraic scaling $x^{-(1+\alpha)}$ corresponding to the solution of the standard $(\lambda=0)$ fractional diffusion equation. Figures 2(b)-2(d) show the propagator $G$ at later times. The superimposed dashed line in Fig. 2(d) is a plot of the function $G_{2, \beta}^{\infty}(z)=z^{a_{1}} \exp \left(-z^{a_{2}}\right)$ with $a_{1}=(\beta-1) /(2-\beta)$ and $a_{2}$ $=2 /(2-\beta)$, which is the asymptotic form of the solution of the subdiffusive $(\alpha=2,0<\beta<1)$ fractional equation [3]. Consistent with the fact that for $\tau \gg 1$ truncation effects are dominant, it is observed that for $\lambda x / \sigma \gg 1, G \rightarrow G_{2, \beta}^{\infty}$.

\section{CONCLUSIONS}

We considered the fluid limit of the continuum time random walk model for the most general jump probability distribution function in the Lévy-Khintchine representation. The resulting integrodifferential equation gives the probability distribution function in the long-time small- $k$ (large scale) limit. The time evolution is governed by a fractional time derivative and the spatial evolution corresponds to the generator for a general Lévy density. As expected, for Lévy stable densities we recover the usual fractional diffusion equation. However, one of the main drawbacks of Lévy stable distributions from the applications point of view is that, due to the algebraic decaying tails, they have divergent moments. In the past, to circumvent this problem, several authors have proposed the use of truncated Lévy densities that lead to finite moments. Of particular interest are exponentially truncated densities in which the algebraic decay of the tails is damped by an exponential factor. These distributions have found widespread applicability in many areas including plasma physics, fluid mechanics, and finance. However, despite their importance, little is known about the role of these distributions in CTRW with memory and in the corresponding fluid limit. Here we addressed this issue and formulated a macroscopic transport equation in terms of truncated fractional derivatives. Because of the truncation, the dynamics ultimately converge to the Gaussian case for $\beta$ $=1$ and to the standard subdiffusive case for $\beta<1$. However, 
(a)

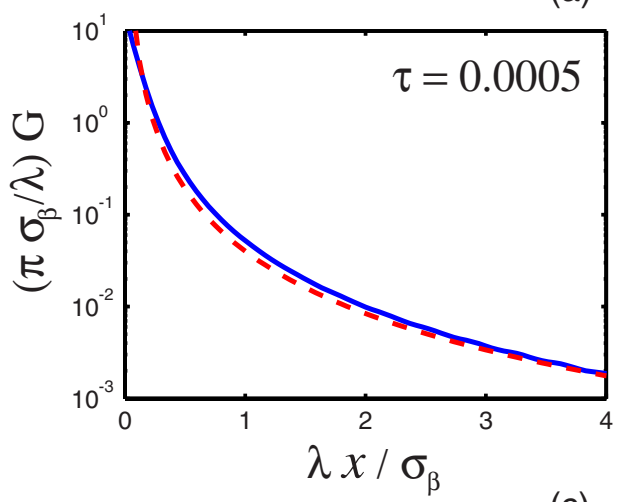

(c)

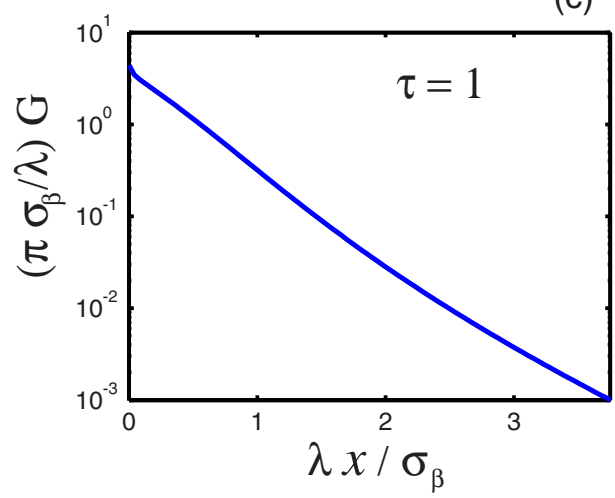

(b)

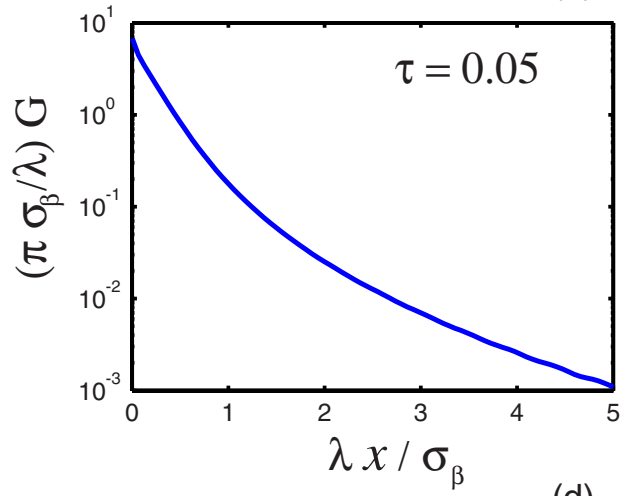

(d)

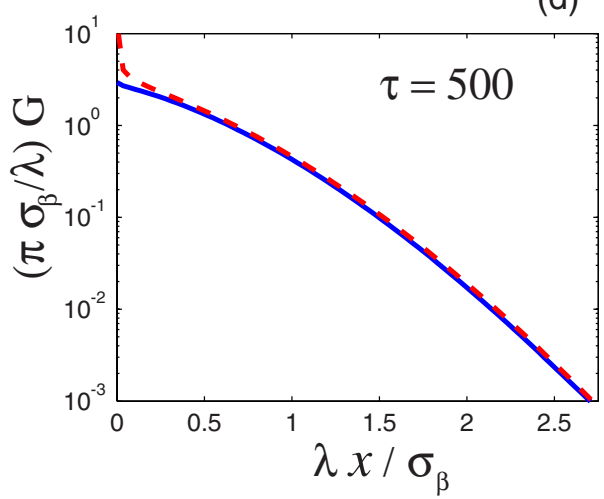

FIG. 2. (Color online) Time evolution of the solution of the truncated $\lambda \neq 0$ fractional diffusion Eq. (23) for a delta function initial condition and $a=\sigma=\theta=0, \alpha=1.25$, and $\beta=0.75$. The dashed lines in panels (a) and (d) correspond to the asymptotic behavior of the solution of the standard $\lambda=0$ fractional diffusion equation for $(\alpha, \beta)=(1.25,0.75)$ and $(\alpha, \beta)=(2,0.75)$, respectively. To ease the comparison at successive times, the axes have been rescaled by the variance in the subdiffusive limit $\sigma_{\beta}=\tau^{\beta / 2}$. A slow transition due to the truncation is observed from the superdiffusive concave algebraic decaying profile in panel (a) to the subdiffusive convex profile in panel (d) with asymptotic stretched Gaussian decay. At the crossover time scale $\tau \sim 1$, the profile shows asymptotically vanishing curvature characteristic of exponential decay.

the key issue is that convergence can be extremely slow and for time scales of interest to applications the process can be considered non-Gaussian but not described by Lévy stable distribution. The proposed truncated fractional diffusion equation describes this intermediate asymptotic regime which cannot be captured by the standard fractional diffusion equation based on Riemann-Liouville derivatives. As an application of the truncated fractional equation we studied the interplay between truncation and memory effects. It was shown that the return probability distribution function exhibits a transition from the Lévy stable scaling $G(0, t) \sim t^{-\beta / \alpha}$ at short times, to $G(0, t) \sim t^{\beta / 2}$ scaling at long times. For $2 \beta / \alpha>1$ this corresponds to a transition from superdiffusive to subdiffusive dynamics. The crossover time to subdiffusive behavior scales as $\tau \sim \lambda^{-\alpha / \beta}$, where $1 / \lambda$ is the truncation length scale. The asymptotic behavior of the propagator
(Green's function) of the truncated fractional diffusion equation exhibits a transition from algebraic decay (characteristic of superdiffusive processes) for $\lambda^{\alpha / \beta} t \ll 1$ to stretched Gaussian decay (characteristic of subdiffusive processes) for $\lambda^{\alpha / \beta} t \gg 1$.

\section{ACKNOWLEDGMENTS}

A.C. acknowledges financial support from the Nuffield Foundation. D.C.N. gratefully acknowledges the hospitality of Birkbeck College of the University of London during the elaboration of this work, and thanks Dr. Raúl Sánchez and Dr. Iván Calvo for helpful comments. Also, D.C.N. acknowledges support from the Oak Ridge National Laboratory, managed by UT-Battelle, LLC, for the U.S. Department of Energy under Contract No. DE-AC05-00OR22725.
[1] E. W. Montroll and G. H. Weiss, J. Math. Phys. 6, 167 (1965).

[2] E. W. Montroll and M. F. Shlesinger, in Nonequilibrium Phenomena II. From Stochastics to Hydrodynamics, edited by J. L. Lebowitz and E. W. Montroll (Elsevier Science Publishers,
Amsterdam, 1984).

[3] R. Metzler and J. Klafter, Phys. Rep. 339, 1 (2000).

[4] R. N. Mantegna and H. E. Stanley, Phys. Rev. Lett. 73, 2946 (1994). 
[5] I. Koponen, Phys. Rev. E 52, 1197 (1995).

[6] M. F. Shlesinger, Phys. Rev. Lett. 74, 4959 (1995).

[7] R. Jha, P. K. Kaw, D. R. Kulkami, J. C. Parikh, and the ADITYA Team, Phys. Plasmas 10, 699 (2003).

[8] B. Dubrulle and J.-Ph. Laval, Eur. Phys. J. B 4, 143 (1998).

[9] R. Bruno, L. Sorriso-Valvo, V. Carbone, and B. Bavassano, Europhys. Lett. 66, 146 (2004).

[10] R. N. Mantegna and H. E. Stanley, Nature (London) 376, 46 (1995).

[11] R. Balescu, Phys. Rev. E 51, 4807 (1995).

[12] B. Ph. van Milligen, R. Sanchez, and B. A. Carreras, Phys. Plasmas 11, 2272 (2004).

[13] D. del-Castillo-Negrete, B. A. Carreras, and V. E. Lynch, Phys. Plasmas 11, 3854 (2004).

[14] D. del-Castillo-Negrete, B. A. Carreras, and V. E. Lynch, Phys. Rev. Lett. 94, 065003 (2005).

[15] D. del-Castillo-Negrete, Phys. Plasmas 13, 082308 (2006).

[16] B. A. Carreras, V. E. Lynch, and G. M. Zaslavsky, Phys. Plasmas 8, 5096 (2001).

[17] I. M. Sokolov, A. V. Chechkin, and J. Klafter, Physica A 336, 245 (2004).

[18] B. Meerschaert and B. Scheffler, Phys. Rev. E 65, 041103
(2002).

[19] M. M. Meerschaert and H.-P. Scheffler, J. Appl. Probab. 41, 623 (2004).

[20] A. Piryatinska, A. I. Saichev, and W. A. Woyczynski, Physica A 349, 375 (2005).

[21] V. M. Kenkre, E. W. Montroll, and M. F. Shlesinger, J. Stat. Phys. 9, 45 (1973).

[22] R. Hilfer and L. Anton, Phys. Rev. E 51, R848 (1995).

[23] E. Scalas, R. Gorenflo, and F. Mainardi, Phys. Rev. E 69, 011107 (2004).

[24] I. Podlubny, Fractional Differential Equations (Academic Press, San Diego, 1999).

[25] A. Saichev and G. M. Zaslasvsky, Chaos 7, 753 (1997).

[26] F. Mainardi, Y. Luchko, and G. Pagnini, Fractional Calculus Appl. Anal. 4, 153 (2001).

[27] K. Sato, Lévy Processes and Infinitely Divisible Distributions (Cambridge University Press, UK, 1999).

[28] G. Samorodnitsky and M. S. Taqqu, Stable non-Gaussian Random Processes (Chapman \& Hall, New York, 1994).

[29] S. G. Samko, A. A. Kilbas, and O. I. Marichev, Fractional Integrals and Derivatives (Gordon and Breach Science Publishers, Amsterdam, 1993). 